\title{
Chemin faisant avec Yves Reuter
}

\author{
Ana Dias-Chiaruttini Université de Lille - Théodile-CIREL 4354 \\ Cora Cohen-Azria Université de Lille - Théodile-CIREL 4354 \\ Un héritage n'étant jamais naturel, on peut hériter plus d'une fois, dans les lieux et à des
moments différents, choisir d'attendre le temps le plus approprié, qui peut être le plus
intempestif - en écrire selon différentes lignées, et signer ainsi sur plus d'une portée.
}

Jacques Derrida, Spectre de Marx, 1993, p. 266.

Les recueils d'hommages se multiplient ces temps-ci dans le domaine de la didactique du français, signe du temps qui passe, signe aussi que la discipline de recherche s'est installée dans le champ universitaire et que le temps des bilans d'étapes est venu. Il nous revient d'identifier les apports qui ont contribué à fonder cette discipline de recherche, à la fois connue (parfois mal-reconnue), identifiée, identifiable, somme toute encore institutionnellement fragile, nécessitant vigilance méthodologique et rigueur théorique. Pour continuer à écrire l'histoire de cette discipline de recherche, il faut sans cesse interroger ses fondements et les choix théoriques qui sont faits, oubliés, écartés...

Yves Reuter a fortement contribué au développement de ce champ par ses travaux, par les concepts qu'il a développés, mais aussi par la création de l'équipe Théodile et le dialogue entre les didactiques qu'il y a permis, loin de tout dogmatisme. Théodile, créée en 1991, est l'une des premières équipes de didacticiens du français à être affiliée aux sciences de l'éducation. En effet, les didacticiens du français étaient - et le sont encore pour une grande partie d'entre eux rattachés à des UFR (Unités de Formation et de Recherche) et à des laboratoires en sciences du langage ou en Lettres. Il faut voir dans ce rattachement de l'équipe Théodile aux sciences de l'éducation, le projet scientifique de son fondateur et de ses collaborateurs de l'époque, d'affirmer l'autonomie de la didactique du français à l'égard des disciplines dites de référence, et de la constituer par là même comme une discipline de référence de la matière français (Halté, 1992). L'appartenance aux sciences de l'éducation, la transversalité des thèmes de recherche retenus, ainsi que la détermination d'Yves Reuter ont contribué au développement d'un collectif de didacticiens de disciplines scolaires diverses et de fait, cela est venu enrichir le projet d'une didactique comparée ou d'un espace de dialogues entre didactiques.

Pour rendre hommage au fondateur de Théodile et à ses travaux, nous avons choisi la forme des Mélanges sans découpage thématique. Nous avons privilégié l'hétérogénéité des contributions laissant percevoir une progression chronologique des thématiques abordées pour embrasser et mettre à l'honneur la diversité et la vitalité des débats, des problématiques et des influences de ses travaux sans aucune quête d'exhaustivité ou de formalisme. L'hétérogénéité n'est cependant ni synonyme ni source d'éclatement ou de disparité, les contributeurs se sont emparés des discussions amorcées, parfois même suscitées par Yves Reuter, pour engager de nouveaux débats et de nouvelles réflexions.

Le titre de l'ouvrage s'ancre dans cette dynamique. Théories - didactiques de la lecture et de l'écriture déplie l'acronyme de l'équipe de recherche fondée par Yves Reuter. Toutefois apparaît une différence significative, le substantif didactique est ici au pluriel, pour souligner la pluralité 
des approches didactiques mises en débat, sans démarche surplombante de l'une ou de l'autre.

À travers les chapitres, les regards portés sur le passé ne sont empreints d'aucun regret, d'aucune nostalgie. Ils sont inscrits dans le présent, et, avec Yves Reuter, ils posent les questions de l'avenir de ce champ de recherche.

Christophe Niewiadomski offre aux lecteurs l'analyse d'un entretien clinique mené avec Yves Reuter. Ce dernier a accepté de se prêter au jeu, sans connaitre d'ailleurs le contexte de cet ouvrage. Leur amitié permet une proximité certaine, rendant la tâche d'écriture parfois plus difficile pour l'auteur. Les qualités techniques de l'interviewer nous permettent d'accéder à une part du discours d'Yves Reuter qui aurait été sans aucun doute difficile à mobiliser dans un autre cadre. Il nous est alors donné à voir un regard singulier porté sur son propre cheminement, les mots posés pour le dire rendant compte de ce parcours individuel fait de recherches et d'entreprises collectives.

André Petitjean retrace le parcours du didacticien au sein de la revue Pratiques où ce dernier s'est engagé très jeune et y est resté fidèle en coordonnant un nombre important de numéros. La succession des thématiques laisse percevoir l'évolution du questionnement de l'enseignant agrégé de Lettres, du formateur d'enseignants puis du chercheur en sciences de l'éducation. Ce faisant, c'est aussi la contribution de la revue Pratiques au développement du champ de recherche de la didactique du français qui se dessine laissant percevoir le rôle de l'engagement militant des premiers chercheurs en didactique du français.

Passionné de romans policiers, Yves Reuter (1989a, 1989b, 1997, 1999a, 2002a, 2002b, 2015) leur consacra quelques-uns de ses écrits. Il a ainsi arpenté et réhabilité les « mauvais genres », questionnant l'institution littéraire et la place de la paralittérature (1986, 1992a, 1992b, 1999b). Ses travaux se sont centrés sur le récit (2001, 2007a) et sur le personnage (1988a, 1988b, 1989a, 1989c, 1990a, 1991, 1992c), une " clé de la compréhension ${ }^{1} »$ du récit dont Yves Reuter rappelait en 1988 que si l'importance des travaux consacrés aux personnages « impose l'impression d'une évidence, elle ne saurait remplacer une définition ». Il propose alors avec ses collègues (Catherine Tauveron et Pierre Glaudes, entre autres) de le considérer comme un marqueur typologique, un organisateur textuel et un lieu d'investissement pour lecteurs et scripteurs.

Dans la continuité de ces travaux, Catherine Tauveron débusque les évidences avec lesquelles certains programmes scolaires, certains manuels scolaires et certains sites professionnels traitent le personnage comme allant de soi sans problématiser les enjeux de cet apprentissage, alors que d'autres proposent des tentatives pour saisir le rôle des personnages, les valeurs qu'ils incarnent, les stéréotypes qu'ils véhiculent, voire dénoncent. Elle propose un cadre d'analyse qui permet de retracer l'évolution du personnage canonique du loup au sein de la littérature de jeunesse, et par là-même l'évolution de ce champ de production littéraire et du lecteur à qui il s'adresse. La revue des titres d'albums dresse un parcours de lecture où l'on suit les métamorphoses du personnage du loup et les stéréotypes qu'il embrasse. Elle avance ainsi un programme didactique de l'étude du personnage du loup renouant avec les premiers travaux d'Yves Reuter.

Pierre Glaudes poursuit par une analyse littéraire de Dingo, le dernier roman d'Octave Mirbeau

\footnotetext{
${ }^{1}$ En référence aux travaux de Catherine Tauveron (1995).
} 
dont le style et le ton ne laisseront indifférent ni le didacticien ni le lecteur espiègle qu'est Yves Reuter, et qui dès ses débuts s'est attelé à penser les activités scolaires pour rendre accessible le sens des textes ${ }^{2}$. Dingo, héros éponyme du roman d'Octave Mirbeau, empruntant son nom à celui du chien de l'écrivain, permet à l'auteur de rompre définitivement avec les codes romanesques du $19^{\mathrm{e}}$ siècle et les conventions génériques. La domestication de Dingo ne le libèrera pas de ses envies sanguinaires et le chien offre au romancier l'occasion d'une satire sociale, d'une démystification des bonnes idées politiques, mondaines, du beau Paris, qui au moment où nous nous apprêtons à célébrer le centenaire de la disparition de Mirbeau est toujours d'actualité.

Ainsi, si les premiers travaux d'Yves Reuter ont porté sur la lecture du récit et les biens littéraires (1990b, 1995), les suivants l'ont amené à formaliser un modèle didactique de l'écriture (Reuter, 1996). En considérant l'écriture comme une pratique sociale dont les enjeux ne se limitent pas à l'acquisition de techniques, Yves Reuter centre sa réflexion sur le sujet didactique et l'image que les élèves construisent d'eux-mêmes selon les situations de production (Delcambre, Reuter, 2002). Pour Yves Reuter, l'analyse " du faire » des élèves ne peut se soustraire du contexte de production, il s'intéresse ainsi aux relations entre les pratiques scolaires et extrascolaires des élèves, notamment à partir des travaux de Penloup (1999) et de Barré-De Miniac (2000).

Ces travaux ont montré combien l'analyse de ce qui se passe hors de l'école est riche d'enseignement pour la didactique du français. Régine Delamotte, qui a beaucoup œuvré à éclairer les apports de la socio-didactique pour mieux appréhender les relations entre les pratiques langagières et les performances scolaires des élèves, revient sur une analyse de l'activité narratrice d'une petite fille, Jeanne, qui raconte à sa mère l'anniversaire de Babar à partir d'un album à des âges différents ( 3 ans et 6 mois puis 4 ans et 6 mois). Cette confrontation des deux récits produits et enregistrés à un an d'écart permet de mettre en évidence les variations, probablement quelques effets des apprentissages liés en partie à la scolarisation de la petite Jeanne, et la façon dont ce dialogue entre fille et mère évolue et laisse place au récit de l'enfant qui prend forme. L'analyse fine des échanges entre la mère et la fille éclaire aussi les jeux de langage et la libre expression de soi, que le récit de l'album permet sans doute et que la situation de communication favorise. Régine Delamotte reprend ainsi des discussions inscrites dans le temps entre la didactique du français et la socio-didactique, entre les pratiques scolaires et extrascolaires, entre Théodile et Lidifra devenu Dysola puis Dylis, entre Yves Reuter, MarieClaude Penloup et elle-même.

Francis Grossmann, quant à lui, revient sur l'une des préoccupations constantes d'Yves Reuter, qu'il a entre autres partagée avec les collègues de l'équipe LIDILEM de Grenoble, en particulier avec Michel Dabène : l'accompagnement à l'écriture de recherche. Une préoccupation qui aujourd'hui encore permet de comprendre la structuration des maquettes de formation de la licence et du master des sciences de l'éducation de Lille 3 et qui rappelle qu'Yves Reuter ne s'est jamais emparé de thématiques médiatisées ou dans l'air du temps. Il a toujours consacré son travail à la compréhension des difficultés que rencontraient les étudiants, les enseignants, les élèves pour saisir les fonctionnements et les dysfonctionnements des enseignements et des apprentissages, et pouvoir ainsi penser et panser l'erreur (2013). La synthèse proposée par Francis Grossmann dresse un panorama des plus intéressants qui éclaire la diversité des

\footnotetext{
${ }^{2}$ Sur ce point, voir, entre autres, les propositions autour d'Angèle qui n'ont rien perdu de leur de leur pertinence, Reuter, 1992.
} 
approches de l'écriture de recherche. La revue des termes pour désigner cet objet de recherche met en lumière les différents champs qui ont contribué à formaliser les questions et leur traitement. Si le propre d'une synthèse est d'être circonscrite, l'enjeu fondamental est de pointer non seulement les apports, mais aussi les écueils et d'apporter de nouvelles propositions qui mettent en valeur les acquis de ces travaux et réorientent les questionnements. Francis Grossmann relève ce défi et propose différentes directions pour poursuivre la recherche, mais aussi pour penser la formation universitaire et doctorale, enjeu fondamental pour écrire l'avenir de notre champ de recherche.

Les chemins de traverse qu'Yves Reuter a parfois, voire souvent, empruntés l'ont amené à interroger les effets de la pédagogie Freinet, éclairant ainsi l'intérêt de prendre en compte les variations pédagogiques dans une réflexion didactique.

La contribution d'Henri Peyronie revient sur la recherche réalisée par différents membres de l'équipe Théodile et ayant donné lieu à l'ouvrage Une École Freinet (Reuter, 2007b). Il met en perspective cette recherche avec différentes études portant sur le devenir des anciens élèves « des pédagogies » Freinet. Cet état des lieux montre, entre autres, les difficultés méthodologiques liées à ce type de recherche. Puisse le dialogue engagé ici donner l'envie à certains lecteurs de prolonger ces questionnements sur le terrain des écoles Freinet.

Les chapitres suivants s'enracinent sur la relecture de différents concepts qu'Yves Reuter a proposés, ou sur les usages des concepts qu'il a discutés. Ce travail conceptuel est le noyau dur, l'épicentre de ses travaux, qui se retrouve dans le projet du Dictionnaire des concepts fondamentaux en didactiques, édité en 2007, puis réédité en 2010 et 2013, qu'il a conçu et dirigé, entre autres, pour aider les étudiants en sciences de l'éducation et les doctorants. Mais il a permis aussi à des didacticiens de disciplines différentes d'y trouver des espaces de débat. Ce dictionnaire est sans aucun doute, avec le séminaire international sur les Méthodes de recherches en didactiques (Perrin-Glorian, Reuter, 2006 ; Lahanier- Reuter, Roditi, 2007 ; Sayac, CohenAzria, 2009 ; Cohen-Azria, Chopin, Orange, 2015), l'un des apports majeurs des travaux de Théodile.

Bernard Schneuwly et Rita Hofstetter s'emparent ici d'un vieux « contentieux » avec Yves Reuter concernant le concept de forme scolaire. Ce dernier a, en effet, souvent discuté les usages de ce concept en didactique, proposant un retour aux sources dans l'entretien mené avec Guy Vincent et présenté dans la revue Recherches en didactiques - Les Cahiers Théodile (Reuter, Courtebras, 2012a, 2012b). Dans ce chapitre, cet outil intellectuel est envisagé en tension entre des usages contrastés, dont les auteurs documentent de façon passionnante les diverses origines et interprétations.

Les chapitres suivants se confrontent au concept de conscience disciplinaire avancé par Yves Reuter et objet d'une recherche de l'équipe Théodile coordonnée par Cora Cohen-Azria, Dominique Lahanier-Reuter et Yves Reuter (2013).

Joël Lebeaume reprend des discussions déjà abordées au moment de la parution de l'ouvrage rendant compte des acquis de la recherche précédemment citée, et à partir du cadre qui est le sien. Il revient sur les apports et les limites de ce concept. Il en rappelle alors la proximité avec ses propres travaux tout en expliquant les raisons pour lesquelles il préfère associer au terme de 
conscience le qualificatif curriculaire plutôt que disciplinaire. Le regard qu'il porte sur ces questionnements et la lecture qu'il fait des travaux menés dans ce champ donnent à voir les racines d'un débat se structurant tant sur les ancrages théoriques que sur les raisons des choix méthodologiques. Gageons que ce débat n'est pas prêt de se terminer et qu'il reste à connaitre l'espace dans lequel Yves Reuter le poursuivra.

Patrick Rayou voit dans ce concept la possibilité de collaboration de recherches entre sociologues et didacticiens. Ainsi, il souligne les « franchissements de frontières » que la conscience disciplinaire permet d'aborder « au prix toutefois de bougés tant dans des formes de didactiques "désincarnées" qu'au sein de sociologies peu capables de s'intéresser au hic et nunc des situations d'apprentissage ". Il montre comment ce concept et les prolongements qui peuvent en être fait permettent d'interroger les espaces périscolaires ainsi que les liens entre le scolaire et l'extrascolaire, et il s'empare du renouvèlement des cadres de réflexion concernant l'éclairage des phénomènes de réussite et d'échec scolaire. Par son approche sociologique, il intègre également une dimension collective dans la construction ou la reconstruction des disciplines, mettant en lumière la contribution des lycéens ou des étudiants dans les contours définitionnels ou matériels de celles-ci.

Martine Rebière et Maryse Jaubert poursuivent la discussion par la confrontation entre conscience disciplinaire et communauté discursive disciplinaire scolaire, en la plaçant dans le contexte de l'équipe bordelaise et l'héritage des travaux de Michel Brossard. Elles évoquent alors les airs de famille existant entre ces deux concepts, dus, entre autres, aux racines communes référées à des disciplines contributoires aux didactiques. Leur analyse comparée de ces deux espaces conceptuels révèle également des différences. Celles-ci concernent, par exemple, l'étude des usages langagiers au sein des disciplines. Ces distinctions peuvent certes être envisagées sous l'angle théorique, mais elles ramènent également aux choix méthodologiques réalisés au sein des recherches menées et auxquelles elles se réfèrent ici.

Enfin, les trois derniers chapitres traitent de la délicate question des enjeux et de la formalisation de la didactique du français, et sans doute abordent-ils par là même l'engagement et le sens de l'œuvre - au sens d'activité - d'Yves Reuter.

Jean-Paul Bronckart soulève la question de la quête d'identité de la didactique et ce faisant il embrasse une série de questionnements qui agence de fines et rigoureuses analyses des réflexions et concepts qui permettent de reconstruire les fondements de la «didactique standard ». Le retour à ce qui fonde l'identité didactique - son projet, sa filiation aux contenus, ses liens avec la pédagogie et surtout avec de nombreux pédagogues, les formes de rupture et de continuité - lui permet de rappeler aussi les impasses épistémologiques, éthiques et idéologiques de l'autoproclamation d'une « théorie anthropologique $d u$ didactique » et de la légèreté des emprunts et des références au concept majeur de cette idéologie : la transposition didactique. Une fois cette clarification apportée, Jean-Paul Bronckart reprend le dialogue amorcé avec Yves Reuter sur les apports de Vygotski aux questions didactiques.

Marie-France Bishop présente les enjeux de la démarche historico-didactique qu'elle a contribué à formaliser et en rappelle les principes méthodologiques. Cette démarche permet non seulement de retracer les emprunts épistémologiques, leurs évolutions et leurs transformations, mais aussi d'éclairer et de comprendre les évolutions historiques et didactiques tissées au cours des périodes. 
C'est au prix de cette rigoureuse reconstruction que l'on peut mieux comprendre ce qui s'enseigne et comment cela s'enseigne aujourd'hui.

Marie-Claude Penloup revient sur l'influence des travaux en didactique du français dans la sphère sociale et dans le champ de la formation des enseignants, en questionnant tout particulièrement l'apport de la socio-didactique. Loin d'un pessimisme démesuré, son analyse rejoint celle que pose Yves Reuter depuis un certain temps constatant le peu de visibilité institutionnelle de la discipline et de ses travaux. Une fois le constat posé, analysé, expliqué, Marie-Claude Penloup dresse toutefois les possibles apports de la socio-didactique et propose un programme didactique qui mettrait moins l'accent sur les manques de savoir- faire des élèves, et qui s'attacherait à rendre compte de tout ce qu'ils savent faire en prenant « acte des variétés qui s'invitent dans les discours sociaux » afin " d'outiller les élèves pour les traiter linguistiquement et socialement parlant ». Une école émancipatrice, en somme !

Le recueil s'achève par une bibliographie des travaux d'Yves Reuter établie par Dominique Lahanier-Reuter qui nous a accompagnées au début de ce projet éditorial et que nous remercions pour tout ce qu'elle a facilité.

Ce parcours n'aborde pas la totalité des questions qu'Yves Reuter ou ses travaux ont pu soulever, cet ouvrage n'en a ni la prétention ni la finalité. Il dessine un chemin singulier fait de rencontres. Il permet de saisir quelques éléments des débats qui ont traversé la didactique du français, le dialogue entre les didactiques, les enjeux de la formation des étudiants, des enseignants. Puisse-til en susciter de nouveaux...

Chemin faisant. Parce que nous faisons le chemin et le chemin nous fait.

\section{Bibliographie}

BARRÉ-DE MINIAC Christine (2000) Le rapport à l'écriture. Aspects théoriques et didactiques, Villeneuve d'Ascq, Presses Universitaires du Septentrion.

COHEN-AZRIA Cora, SAYAC Nathalie (éds) (2009) Questionner l'implicite. Les méthodes de recherche en didactique(s) (3), Villeneuve d'Ascq, Presses Universitaires du Septentrion.

COHEN-AZRIA Cora, LAHANIER-REUTER Dominique, REUTER Yves (dir.) (2013) Conscience disciplinaire. Les représentations de disciplines à la fin de l'école primaire, Rennes, Presses Universitaires de Rennes.

COHEN-AZRIA Cora, CHOPIN Marie-Pierre, ORANGE-RAVACHOL Denise (éds) (2015) Questionner l'espace. Les méthodes de recherche en didactiques (4), Ville- neuve d'Ascq, Presses Universitaires du Septentrion.

DELCAMBRE Isabelle, REUTER Yves (dir.) (2002) Pratiques, $\mathrm{n}^{\circ} 113-114$, Images du scripteur et rapports à l'écriture.

GLAUDES Pierre, REUTER Yves (dir.) (1991) Personnage et histoire littéraire, Toulouse, Presses Universitaires du Mirail. 
HALTÉ Jean-François (1992) La didactique du français, Paris, Presses Universitaires de France, Que sais-je?

LAHANIER-REUTER Dominique, RODITI Éric (éds) (2007) Questions de tempo- ralité, les méthodes de recherche en didactiques (2), Villeneuve d'Ascq, Presses Univer- sitaires du Septentrion.

PENLOUP Marie-Claude (1999) L'écriture extraordinaire des collégiens. Des constats aux perspectives didactiques, Paris, ESF.

PERRIN-GLORIAN Marie-Jeanne, REUTER Yves (dir.) (2006) Les méthodes de recherche en didactiques, Villeneuve d'Ascq, Presses Universitaires du Septentrion.

REUTER Yves (dir.) (1986) Pratiques, $\mathrm{n}^{\circ}$ 50, Les paralittératures. REUTER Yves (dir.) (1988a) Le personnage dans les récits. Cahiers de Recherches en Didactique du Français $\mathrm{n}^{\circ} 2$, Université de Clermont-Ferrand, CRDP.

REUTER Yves (dir.) (1988b) Pratiques, nº 60, Le personnage.

REUTER Yves (dir.) (1989a) Le roman policier et ses personnages, Paris, Presses Universitaires de Vincennes.

REUTER Yves (1989b) Éléments pour une typologie des romans policiers, Tapis Franc, n $^{\circ} 2$, 77-97.

REUTER Yves (1989c) Personnages et conflits dans le récit. Éléments de réflexion, Cahiers de Recherches en Didactique du Français, $\mathrm{n}^{\circ} 3$, Personnages et histoires, CRDP. Université de Clermont-Ferrand II, 157-172.

REUTER Yves (1990a) Le roman sentimental : système des personnages et circulation sociale de la thématique amoureuse, dans Ellen CONSTANS (dir.) Trames, Le roman sentimental, Limoges, Presses universitaires de Limoges, 209-223.

REUTER Yves (1990b) Définir les biens littéraires ?, Pratiques, n 67, 5-14.

REUTER Yves (éd.) (1992a) Personnage et roman policier, Saint Denis, Presses Universitaires de Vincennes.

REUTER Yves (1992b) Littérature/Paralittératures : classements et déclassements, dans Jacques La Mothe : Actes du Colloque Les mauvais genres, Liège, Éditions du C.L.P.F., Les Cahiers des Paralittératures $\mathrm{n}^{\circ}$ 3, 37-47.

REUTER Yves (1992c) Comprendre, interpréter, expliquer des textes en situation scolaire. « À propos d'Angèle », Pratiques, n 76, 7-25.

REUTER Yves (1994) Places et fonctions du savoir dans les romans à suspense, dans Jean-Marie GRAITSON, (éd.) Agatha Christie et le roman policier d'énigme, Actes du $5^{\mathrm{e}}$ colloque international de Chaudfontaine, Liège, Éditions du CEFAL, 125-140. 
REUTER Yves (1995) Pour une didactique de la littérature : la nécessaire définition des biens littéraires, dans Christian POSLANIEC, (éd.) Littérature et jeunesse, Actes du colloque ParisINRP, 23-24-25 mars 1993, Paris, INRP, 129-145.

REUTER Yves (1996) Enseigner et apprendre à écrire. Construire une didactique de l'écriture, Paris, ESF.

REUTER Yves (1997) Le roman policier, Paris, Nathan, coll. «128».

REUTER Yves (1999b) Corpus littéraires et paralittératures, dans Lecteurs, Littérature, Enseignement, Actes du XI e congrès de l'AFEF, Le français d'aujourd'hui, Hors série, 113-118.

REUTER Yves (2002a) De quelques modalités de la mise en jeu des savoirs dans le roman policier, dans Claude AMBROISE et Georges TYRAS, (éds) Violence politique et écriture de l'élucidation dans le bassin méditerranéen, Grenoble, Tigre/ Novecento, Hors série, n 21-31.

REUTER Yves (2002b) Roman policier et enseignement du français, Page des libraires, Page Éducation, hors série, septembre 2002, 30-33.

REUTER Yves (dir.) (2007a) Pratiques, $\mathrm{n}^{\circ}$ 133-134, Récits et disciplines scolaires. REUTER Yves (dir.) (2007b) Une école Freinet. Fonctionnements et effets d'une pédagogie alternative en milieu populaire, Paris, L'Harmattan.

REUTER Yves (dir.) (2007/2010/2013) Dictionnaire des concepts fondamentaux des didactiques, Bruxelles, De Boeck. REUTER Yves (2013) Panser l'erreur. De l'erreur au dysfonctionnement, Villeneuve d'Ascq, Presses Universitaires du Septentrion.

REUTER Yves (2015) L'étrange disponibilité du roman policier, Revue critique de fixxion française, $\quad \mathrm{n}^{\circ} \quad 10, \quad 4-12 \quad: \quad$ http://www.revue-critique-de-fixxion-francaisecontemporaine.org/rcffc/article/view/ fx10.02/979.

REUTER Yves, COURTEBRAS Bernard (2012a) La forme scolaire : débats et mises au point (première partie) ; entretien avec Guy Vincent, Recherches en Didactiques, $\mathrm{n}^{\circ} 13,109-135$.

REUTER Yves, COURTEBRAS Bernard (2012b) La forme scolaire : débats et mises au point (seconde partie) ; entretien avec Guy Vincent, Recherches en Didactiques, ${ }^{\circ} 14,127-143$.

REUTER Yves, TAUVERON Catherine (dir.) (2001) Repères, $\mathrm{n}^{\circ} 21$, Diversité narrative. TAUVERON Catherine (1995) Le personnage. Une clef pour la didactique du récit à l'école élémentaire, Neuchâtel, Delachaux \& Niestle. 